\title{
CONVERGENCE OF LAPLACIANS ON SMOOTH SPACES TOWARDS THE FRACTAL SIERPIŃSKI GASKET
}

\author{
OLAF POST AND JAN SIMMER
}

Abstract. The purpose of this article is to prove that - under reasonable assumptions - the canonical energy form on a graph-like manifold is quasi-unitarily equivalent with the energy form on the underlying discrete graph. Then we will apply this to approximate the standard energy form on the Sierpiński gasket by a family of energy forms on suitable graph-like manifolds.

Mathematics subject classification (2010): 28A80, 05C75, 47A58. forms.

Keywords and phrases: Sierpiński gasket, approximation by smooth manifolds, convergence of energy

\section{REFERENCES}

[1] C. ANNÉ AND O. Post, Wildly perturbed manifolds: norm resolvent and spectral convergence, (accepted for publication in J. Spectr. Theory) (2020).

[2] T. Berry, S. M. Heilman, And R. S. StRichartz, Outer approximation of the spectrum of a fractal Laplacian, Experiment. Math. 18 (2009), 449-480.

[3] A. Blasiak, R. S. StRichartz, AND B. E. UĞURCAn, Spectra of self-similar Laplacians on the Sierpinski gasket with twists, Fractals 16 (2008), 43-68.

[4] I. Chavel, Isoperimetric inequalities, Cambridge Tracts in Mathematics, vol. 145, Cambridge University Press, Cambridge, 2001, Differential geometric and analytic perspectives.

[5] T. Kato, Perturbation theory for linear operators, Springer-Verlag, Berlin, 1966.

[6] J. Kigami, Analysis on fractals, Cambridge Tracts in Mathematics, vol. 143, Cambridge University Press, Cambridge, 2001.

[7] A. Khrabustovskyi And O. Post, Operator estimates for the crushed ice problem, Asymptot. Anal. 110 (2018), 137-161.

[8] U. Mosco And M. A. Vivaldi, Layered fractal fibers and potentials, J. Math. Pures Appl. (9) 103 (2015), 1198-1227.

[9] O. Post, Spectral convergence of quasi-one-dimensional spaces, Ann. Henri Poincaré 7 (2006), 933 973.

[10] O. Post, Spectral analysis on graph-like spaces, Lecture Notes in Mathematics, vol. 2039, Springer, Heidelberg, 2012.

[11] O. Post And J. SimmeR, Approximation of fractals by discrete graphs: norm resolvent and spectral convergence, Integral Equations Operator Theory 90 (2018), 90:68.

[12] O. Post AND J. Simmer, Approximation of fractals by manifolds and other graph-like spaces, (accepted for publication in Mathematische Nachrichten) (2020).

[13] O. POST AND J. SIMMER, Quasi-unitary equivalence and generalised norm resolvent convergence, Rev. Roumaine Math. Pures Appl. 64 (2019), 2-3, 373-391.

[14] O. Post AND J. Simmer, Convergence of finite dimensional approximations of magnetic Laplacians on finitely ramified fractals, preprint (2019).

[15] M. ReEd AND B. Simon, Methods of modern mathematical physics I: Functional analysis, Academic Press, New York, 1980.

[16] R. S. Strichartz, Differential equations on fractals, Princeton University Press, Princeton, NJ, 2006. 